\title{
Impacto da cobertura e da qualidade da atenção básica nas internações por condições sensíveis à Atenção Primária em Sergipe de 2010 a 2019
}

Impact of coverage and quality of primary care on admissions for Primary Care-sensitive conditions in Sergipe of 2010 to 2019

Impacto de la cobertura y la calidad de la Atención Primaria en los ingresos por patologías sensibles a la atención primaria en Sergipe de 2010 a 2019

\author{
Annie Ferraz de Queiroz \\ ORCID: https://orcid.org/0000-0002-9952-9291 \\ Universidade Federal de Sergipe, Brasil \\ E-mail: annieferque@gmail.com \\ Rynat Dasaev Oliveira Chagas \\ ORCID: https://orcid.org/0000-0002-4509-0623 \\ Universidade Federal de Sergipe, Brasil \\ E-mail: rynat.chagas@gmail.com \\ Helenita Costa Quadros \\ ORCID: https://orcid.org/0000-0002-7191-4809 \\ Instituto Gonçalo Moniz, Fundação Oswaldo Cruz, Brasil \\ E-mail: helenita_quadros@hotmail.com \\ Marco Antonio Prado Nunes \\ ORCID: https://orcid.org/0000-0001-5244-5843 \\ Universidade Federal de Sergipe, Brasil \\ E-mail: nunes.ma@outlook.com \\ João Batista Cavalcante Filho \\ ORCID: https://orcid.org/0000-0001-8642-9868 \\ Universidade Federal de Sergipe, Brasil \\ E-mail: joao.cavalcante@academico.ufs.br
}

\begin{abstract}
Resumo
Internações por Condições Sensíveis à Atenção Primária (ICSAP) é um indicador usado para avaliar o desempenho do sistema de saúde e da Atenção Básica (AB). Esse estudo teve como objetivo analisar as tendências dessas internações, no Estado de Sergipe, nos anos de 2010 a 2019, além de descrever a influência da cobertura da AB e da participação dos municípios no Programa Nacional de Melhoria do Acesso e da Qualidade da Atenção Básica (PMAQ) sobre o indicador. Foi realizado um estudo ecológico das séries temporais das ICSAP por meio da análise de regressão linear segmentada e realizada correlação com a tendência da cobertura da AB e com a participação no PMAQ. Foi observado uma estabilidade tanto das taxas de distribuição das ICSAP como da cobertura da AB durante a década analisada, sendo que não apresentaram correlação entre as duas variáveis. Ademais, uma maior participação no PMAQ e um bom rendimento no programa mostraram-se capazes de proporcionar menores taxas de ICSAP e estagnação das taxas ao longo do período analisado. Com isso, nosso estudo sugere que, como indicador da qualidade da AB, o ICSAP deve ser utilizado concomitante a uma avaliação de outros aspectos histórico-estruturais e que o PMAQ atingiu o seu objetivo em aumentar o acesso e a qualidade da AB no Estado de Sergipe.

Palavras-chave: Atenção primária à saúde; Estratégia saúde da família; Avaliação em saúde; Reembolso de incentivo.

Abstract

Hospitalizations for Sensitive Conditions to Primary Care (ICSAP) is an indicator used to assess the performance of the health system and Primary Care (AB). This study aimed to analyze the trends of these hospitalizations, in the State of Sergipe, from 2010 to 2019, in addition to describing the influence of coverage of AB and the participation of municipalities in the National Program for Improving Access and Quality of Primary Care (PMAQ) on the indicator. An ecological study of the ACSC time series was carried out by means of segmented linear regression analysis and correlation with the $\mathrm{AB}$ coverage trend and with the participation in the PMAQ. A stability was observed both in the ICSAP distribution rates and in the $\mathrm{AB}$ coverage during the analyzed decade, with no correlation between the two variables. Furthermore, a greater participation in the PMAQ and a good performance in the program proved to be capable of providing lower ICSAP rates and rate stagnation throughout the analyzed period. Thus, our study suggests
\end{abstract}


that, as an indicator of the quality of AB, the ICSAP should be used concomitantly with an evaluation of other historical-structural aspects and that the PMAQ achieved its objective of increasing the access and quality of $\mathrm{AB}$ in the State of Sergipe.

Keywords: Primary health care; Family health strategy; Health evaluation; Pay for performance.

\section{Resumen}

Las hospitalizaciones para condiciones sensibles a la atención primaria (ICSAP) son un indicador utilizado para evaluar el desempeño del sistema de salud y la atención primaria (AB). Este estudio tuvo como objetivo analizar las tendencias de estas hospitalizaciones, en el estado de Sergipe, de 2010 a 2019, además de describir la influencia de la cobertura de $\mathrm{AB}$ y la participación de los municipios en el programa nacional para mejorar el acceso y la calidad de la atención primaria (PMAQ) en el indicador. Un estudio ecológico de la serie de tiempo ICSAP se llevó a cabo mediante análisis y correlación de regresión lineal segmentada con la tendencia de la cobertura AB y con la participación en la PMAQ. Se observó una estabilidad tanto en las tasas de distribución de ICSAP como en la cobertura AB durante la década analizada, sin correlación entre las dos variables. Además, una mayor participación en la PMAQ y un buen desempeño en el programa demostró ser capaz de proporcionar tasas de ICSAP más bajas y tasar el estancamiento a lo largo del período analizado. Por lo tanto, nuestro estudio sugiere que, como indicador de la calidad de $\mathrm{AB}$, el ICSAP debe usarse concomitantemente con una evaluación de otros aspectos históricosestructurales y que la PMAQ logró su objetivo de aumentar el acceso y la calidad de AB en el estado de Sergipe.

Palabras clave: Atención primaria de salud; Estrategia de salud familiar; Evaluación en salud; Reembolso de incentivo.

\section{Introdução}

A Atenção Primária à Saúde (APS) é o primeiro contato preferencial às redes de atenção à saúde e alia um conjunto de ações de promoção, prevenção, tratamento e reabilitação, que interfere diretamente no desenvolvimento da saúde comunitária, melhorando os indicadores de saúde (Ribeiro, Araujo Filho, \& da Rocha, 2019). A qualidade da APS, bem como seu acesso, são elementos essenciais para sistemas de saúde eficazes. Apesar de incluir serviços múltiplos e de difícil mensuração, diversos autores relacionaram uma maior qualidade e um maior acesso à APS a um menor risco de internações por condições sensíveis, devido a sua ação oportuna em evitar ou em reduzir a frequência dessas hospitalizações (Agabiti, et al., 2009; Rasella, Harhay, Pamponet, Aquino, \& Barreto, 2014; Rasmussen, et al., 2007).

Internações por Condições Sensíveis à Atenção Primária (ICSAP) é um dos indicadores usados para monitorar o desempenho do sistema de saúde em diversos países, sendo considerado um indicador indireto da APS. Seu uso traz a ideia de que a falha no acesso à APS de qualidade expõe a população a hospitalizações que poderiam ter sido evitadas caso certas comorbidades fossem identificadas precocemente, podendo reduzir a gravidade ou o aparecimento de complicações (Kim, Park, Yoon, \& Kim, 2019; Macinko, et al., 2011).

As propostas dos estudos sobre ICSAP, nos países onde o acesso à APS é universal e gratuito, direcionam-se para avaliação da qualidade desses sistemas de saúde (Rosano, et al., 2012). Entretanto, essas medidas têm sido pouco utilizadas para mensurar o desempenho de países de média e baixa renda. Avaliar esse indicador no Brasil reveste-se de importância devido ao processo contínuo do desenvolvimento do sistema nacional de saúde que ocorre desde 1988 (Macinko, Harris, \& Rocha, 2017).

Outra utilidade do indicador abrange a identificação de áreas prioritárias em termos de gestão, prestação de serviços, infraestrutura local, investimento econômico e intervenções da APS (Rosano, et al 2012). Compreender o impacto da APS nas hospitalizações é importante para o gerenciamento, uma vez que as internações são um grande gerador de custos em saúde (Bastos, Menzies, Hone, Dehghani, \& Trajman, 2017). O governo brasileiro custeia cerca de 80\% de todas as hospitalizações, que consomem aproximadamente 70\% de todos os gastos nacionais com saúde (Macinko, et al., 2011).

A interpretação das ICSAP não é uniforme, variando entre países, sistemas de saúde, áreas geográficas e grupos populacionais diferentes (Rosano, et al., 2012). Como a maioria dos estudos já existentes sobre ICSAP abrange o sudeste do país, a análise de outras regiões brasileiras torna-se necessária devido as desigualdades socioespaciais existentes (Pedraza \& 
Araujo, 2017). Por isso, faz-se necessário analisar a evolução temporal das ICSAP, no Estado de Sergipe, onde existe carência de tal abordagem.

Ainda considerando a qualidade e a acessibilidade da APS, o Ministério da Saúde (MS) instituiu o Programa Nacional de Melhoria do Acesso e da Qualidade da Atenção Básica (PMAQ), que possui um conjunto de estratégias de qualificação, de acompanhamento e avaliação do trabalho das equipes de saúde (Portaria $\mathrm{n}^{\circ}$ 1.654, 2011). O PMAQ representou o primeiro esquema de pagamento por desempenho do país. Durante seus três ciclos, forneceu incentivos financeiros por meio de indicadores de estrutura, processo e resultado, visando a otimização da APS. Espera-se efeitos diretos na saúde da população caso esse aperfeiçoamento ocorra, sendo o indicador ICSAP potencialmente capaz de mensurar essa realidade (Russo, et al., 2021; Soares \& Ramos, 2020)

Diante da relevância da compreensão do indicador ICSAP e sua evolução com o tempo, esse estudo se propõe a analisar as tendências dessas internações, no Estado de Sergipe, de 2010 a 2019, além de avaliar a influência da variabilidade da cobertura da atenção básica e da participação no PMAQ sobre o indicador. Portanto, este estudo se apresenta tanto como um meio de compreensão do cenário atual, como também uma forma de analisar o impacto das políticas de saúde vigentes no período nas ICSAP.

\section{Metodologia}

Foi realizado um estudo ecológico das séries temporais das ICSAP ocorridas no Estado de Sergipe, reunindo suas sete regiões de saúde (Aracaju, Estância, Lagarto, Itabaiana, Nossa Senhora do Socorro, Nossa Senhora da Glória e Propriá) no período de $1^{\circ}$ de janeiro de 2010 a 31 de dezembro de 2019. Essas regiões são referências da Secretaria de Estado da Saúde de Sergipe (SES-SE), presentes no plano estadual de saúde 2016 - 2019 (Governo do Estado de Sergipe, 2016)

As informações sobre as ICSAP foram extraídas do banco do Sistema de Informações Hospitalares (SIH) do Departamento de Informação e Informática do SUS (DATASUS), tabuladas com auxílio do programa TabWin. O ano de ocorrência da internação na Autorização de Internação Hospitalar (AIH) foi usado para definir a temporalidade do evento, foram excluídos os não moradores do estado nessa análise.

Foi definido como causa de internação o diagnóstico principal que se encontrava discriminado em código de Classificação Estatística Internacional de Doenças e Problemas relacionados à Saúde - Décima Revisão (CID- 10), classificadas como ICSAP, de acordo com a Portaria no 221/2008 do MS.

As taxas brutas de ICSAP foram calculadas por meio da razão entre o número de ICSAP e a população de referência para o período multiplicada por 1.000. O cálculo da população foi pelas estimativas elaboradas pelo MS. As taxas foram padronizadas pelo método direto, considerando a proporção da população mundial estimada pela OMS de 2000 a 2025 (Ahmad, Boschi-pinto, \& Lopez, 2001).

Os dados foram agrupados por região de saúde, e a análise descritiva foi executada com o programa BioEstat 5.0. Para identificar mudanças significativas na tendência das variáveis com o tempo e estimar a variação anual percentual, foi realizada a análise de regressão linear segmentada, com o uso do software Joinpoint versão 4.8.8. O nível de significância adotado foi de $5 \%$. Classificou-se a tendência da série como estabilidade $(p>0,05)$, redução $(p<0,05$ e coeficiente da regressão negativo) ou crescimento ( $\mathrm{p}<0,05$ e coeficiente da regressão positivo).

Para avaliar a cobertura da $\mathrm{AB}$, foi utilizado o banco de dados disponibilizados para consulta pública no e-Gestor Atenção Básica - Informação e Gestão da Atenção Básica. A partir disso, foram coletadas as coberturas mensais e realizada a média anual de cada região de saúde. Por meio do software Joinpoint versão 4.8.8, também foi verificado a tendência da cobertura $\mathrm{AB}$ e a sua variação anual percentual. 
Posteriormente, realizou-se a correlação de Spearman, com o Software BioEstat (versão 5.3, Instituto de Desenvolvimento Sustentável Mamirauá, Belém, Pará, Brasil), para testar a correlação das variáveis acima com as taxas de ICSAP. Como classificação utilizou-se o seguinte parâmetro com base no módulo do coeficiente de correlação (r): correlação fraca quando $0<\mathrm{r}<0,4$; moderada quando $0,4 \leq \mathrm{r} \leq 0,7$ e forte quando $0,7<\mathrm{r}<1,020$. Foram consideradas correlações com significância estatística as que apresentaram $\mathrm{p} \leq 0,05$ e grau de correlação moderado ou forte.

Para analisar os efeitos do PMAQ sobre as ICSAP, foram utilizadas as estratégias de mensurar as equipes participantes do programa e o repasse financeiro, sendo as unidades de análise os municípios de Sergipe participantes do segundo ciclo do programa. A segunda rodada do programa foi escolhida para a análise devido ao primeiro ciclo possuir a condição limitante de apenas 50\% das ESF de um município poder participar do programa, sendo que nas rodadas seguintes, essa restrição não se aplicou. Além disso, como é esperado que a participação no PMAQ proporcione mais prevenção e melhor tratamento no nível da APS, a temporalidade do segundo ciclo, no recorte do presente estudo, é estratégica para avaliar tanto o contexto prévio anterior a adesão, quanto seus efeitos ao longo dos anos consecutivos. As equipes participantes do PMAQ que foram classificadas como 'insatisfatórias' ou 'desclassificadas' foram excluídas do banco de dados, já que não foram expostas ao esquema do PMAQ durante toda a rodada.

No primeiro momento, os municípios foram classificados em três grupos de acordo com a porcentagem de equipes que aderiram ao programa em relação ao número total de ESF de cada município no ano de 2015. O primeiro grupo englobando os municípios com 100\% de participação das equipes, o segundo os que variaram de 50 a $100 \%$ e o terceiro os que possuíam menos de $50 \%$ de participação.

Posteriormente, foram colhidos os repasses financeiros mensais de cada município do Estado de Sergipe no mesmo ciclo. Como os valores são variáveis de acordo a nota obtida no programa, espera-se refletir melhor a qualificação dos municípios diante o PMAQ. Para uniformização dos dados, foram coletados sempre os valores da última lista lançada do programa com o repasse financeiro e dividido pela população do município, gerando valores de repasse per capita. A partir dessa coleta, foi realizada a média do repasse financeiro estadual e separado os municípios em dois grupos: aqueles que se encontravam acima da média e aqueles que ficaram abaixo da média.

Todos os dados do PMAQ foram obtidos por meio do da Secretaria de Atenção Primária à Saúde. Com essas medidas, foram aplicados o teste ANOVA de uma via, seguido do pós-teste de Bonferroni para comparação entre os grupos, e as figuras foram geradas a partir do software Graph Pad 5.0.1, sendo considerado significativo valores de $p \leq 0,05$.

Esse trabalho foi submetido ao Comitê de Ética em Pesquisa envolvendo Seres Humanos da Universidade Federal de Sergipe (CEP-HU/UFS), visando atender às recomendações da Resolução nº 466/12 do Conselho Nacional de Saúde (Brasil, 2013), tendo sido aprovado, com número CAAE 16850219.7.0000.5546.

\section{Resultados e Discussão}

\subsection{Evolução ICSAP}

No período de 2010 a 2019, houve 926.564 internações no Estado, e a população variou de 2108297 para 2298696 habitantes. Durante essa década, o total de ICSAP foi de 129.836, representando 14,01\% do total de internações do Estado de Sergipe. Estudos nacionais apontam uma variação de ICSAP de 12,1 a 13,9\%, em relação ao número total de internações dos municípios brasileiros em período semelhante, mostrando que o Estado de Sergipe se encontra com variação dentro do esperado no país (Pinto, Mendonça, Rehem, \& Stelet, 2019).

As ICSAP são monitoradas rotineiramente pelos sistemas de saúde como um indicador de desempenho no que se refere aos cuidados primários de AB. A taxa de distribuição das ICSAP, no Estado de Sergipe, manteve-se estável durante a 
década analisada, com variação percentual anual de crescimento não significativo de $1,4 \%$, sendo a taxa ICSAP estadual de 5,60 por mil habitantes (Tabela 1).

A taxa encontrada foi semelhante ao estudo de Justo, Bastos e Nedel (2020), que encontraram uma taxa global de 5,8 ICSAP por mil habitantes no Estado de Sergipe durante a década de 2008 a 2017. Além dessa equiparação a nível regional, os dados de estabilidade encontrados no Estado se aproximam da realidade de diversas capitais dos estados brasileiros. Pinto et al (2019) analisaram o período de 2009 a 2018 do Distrito Federal e do conjunto das 27 unidades de federação do País e também foi visto uma tendência de estabilidade das taxas de ICSAP.

Dentre as regiões de saúde, as maiores taxas de internações são de Propriá e de Aracaju, com taxas respectivamente de 9,17 e 8,98, apresentando-se maiores que a taxa do Estado de Sergipe (Tabela 1).

$\mathrm{Na}$ análise da tendência das taxas, foi verificado que as ICSAP se mantiveram estáveis em cinco das sete regiões de saúde. Na região de Nossa Senhora de Socorro, foi identificado tendência crescente significativa das taxas de ICSAP nos anos de 2010 a 2017. Após esse período, ocorreu um ponto de inflexão e tendência decrescente não significativa nos anos posteriores, englobando 2017 a 2019. Na região de Itabaiana, a tendência foi de redução das taxas de ICSAP entre 2010 e 2012, com posterior tendência de crescimento das taxas de 2012 a 2019 (Tabela 1).

Tabela 1: Distribuição das medianas e tendência das taxas ICSAP em Sergipe e nas regiões de saúde de 2010 a 2019.

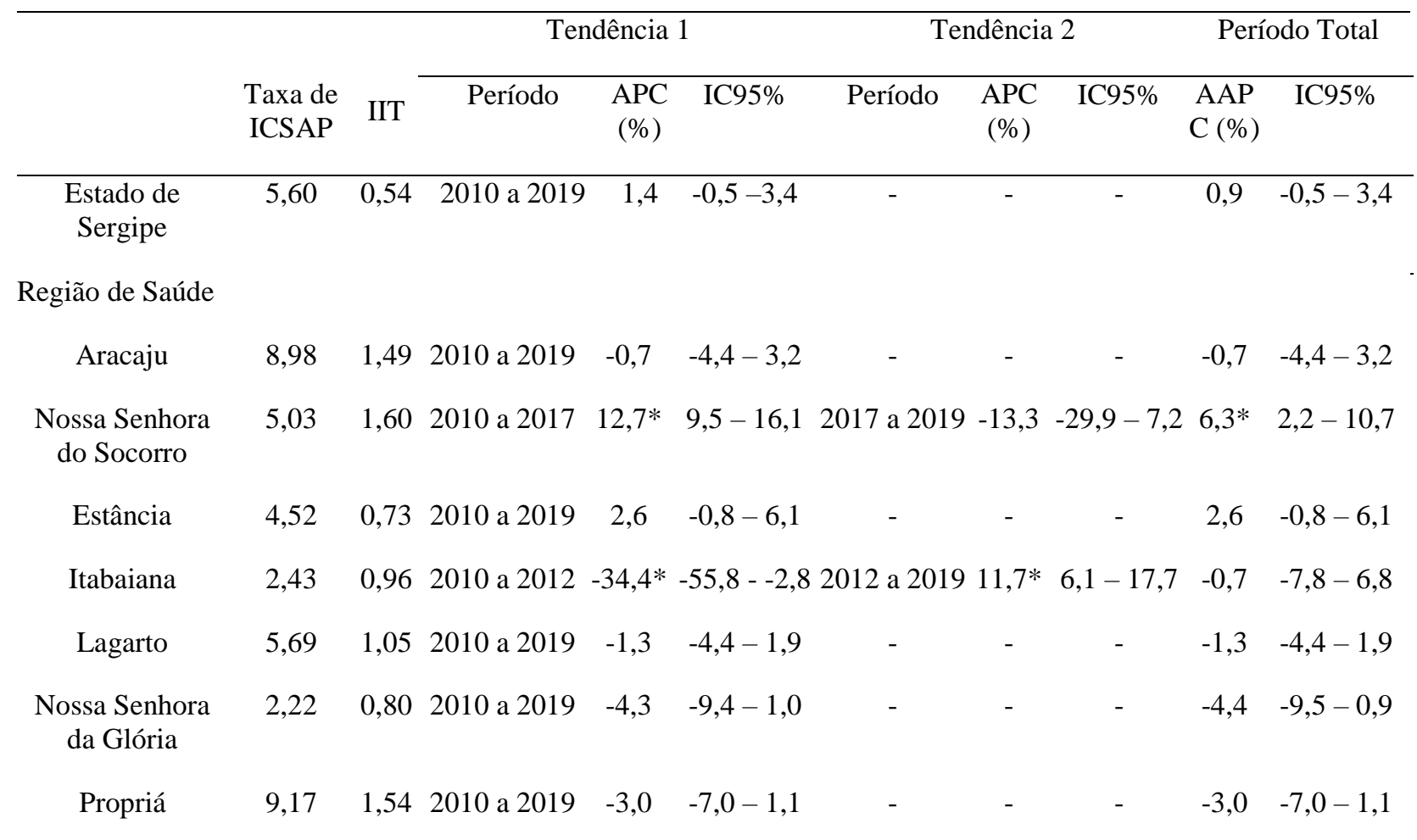

Nota: IIT: Intervalo Interquartil; APC: annual percent change; AAPC: average annual percent change; IC95\%: intervalo de confiança de 95\%; *resultados com significância estatística. Fonte: Autores.

Justo, Bastos e Nedel (2020) analisaram a tendência do ICSAP em Sergipe, no período de 2008 a 2017, e descreveram uma curva decrescente no período de 2008 a 2011, com pouca oscilação de 2011 a 2014, mantendo uma certa estabilidade, com posterior ascensão de 2014 a 2017. Os autores propuseram como explicação para tais resultados uma série de ações realizadas no Brasil para promover a $\mathrm{AB}$ desde 2006, com posterior mudança a partir de 2014. Com isso, o indicador melhorou no período inicial do estudo, com a introdução de políticas públicas que fortaleceram a APS, e sofreu piora à medida que eram 
retiradas ou negligenciadas. Esses motivos também podem explicar a estabilidade do presente estudo, já que o recorte temporal foi posterior a 2008, sendo analisado um período próximo a mudança das políticas públicas no país.

\subsection{Cobertura AB}

A cobertura da AB variou, no Estado de Sergipe, de 90,98\% em 2010 a 88,55\% em 2019, com valor de mediana de 88,59\%. Com a análise da regressão, essa variável teve tendência de estabilidade durante o período. Essa estabilidade da cobertura AB também foi observada em um estudo realizado no Estado de São Paulo. Inicialmente, foi visto uma tendência de ampliação do Programa de Saúde da Família nos anos iniciais do estudo, de 2000 a 2006, entretanto nos posteriores três anos da pesquisa, houve uma tendência de estabilização da cobertura, englobando os anos de 2007 a 2009 (Sala \& Mendes, 2011).

A região de saúde de Aracaju apresentou tendência de redução da porcentagem de cobertura da $\mathrm{AB}$, variando de 97,61\% em 2010 a 79,66\% em 2019. As regiões de Estância, Itabaiana e Lagarto tiveram aumento da cobertura nesses dez anos, sendo a maior variação encontrada em Lagarto. Nessa região, a cobertura AB variou de 60\% em 2010 para 85,51\% em 2019 (Tabela 2).

Apesar das demais regiões permaneceram estáveis durante esses anos, a maior mediana da cobertura AB foi encontrada nas regiões de Nossa Senhora do Socorro, com 99,28\% de cobertura, de Propriá, com 99,11\%, seguidas de Nossa Senhora da Glória, com 93,81\%, todas com cobertura acima da mediana estadual (Tabela 2).

Embora as hospitalizações sensíveis à atenção primária possam tender a diminuir com o aumento da cobertura $\mathrm{AB}$, isso pode não ser aplicável caso os serviços sejam insuficientes ou as condições sensíveis à atenção primária estejam em um estágio avançado. Além disso, o efeito para doenças crônicas como hipertensão ou diabetes provavelmente envolve longos períodos, o que deve ser modificado em um período maior do que o analisado nesse recorte de tempo. Por sua vez, as taxas de hospitalização também dependem da disponibilidade de leitos para internação, suporte financeiro para garantir a universalidade e a integralidade da atenção primária, capacitação de profissionais, disponibilidade e localização dos serviços (Bastos, et al., 2017; Ribeiro, et al., 2019).

Tabela 2: Distribuição das medianas e tendências da cobertura AB em Sergipe e nas regiões de saúde de 2010 a 2019.

\begin{tabular}{ccccc}
\hline & \multicolumn{4}{c}{ Cobertura da Atenção Básica } \\
\hline & Mediana & IIT & APC (\%) & IC95\% \\
\hline Estado de Sergipe & 88,59 & 1,84 & $-0,1$ & $-0,5-0,2$ \\
Região de Saúde & & & & \\
Aracaju & 86,99 & 10,05 & $-2,4^{*}$ & $-3,3--1,6$ \\
Nossa Senhora do Socorro & 99,28 & 0,14 & $-0,1$ & $-0,1-0,0$ \\
Estância & 94,16 & 5,77 & $1,1^{*}$ & $0,4-1,7$ \\
Itabaiana & 88,61 & 2,39 & $0,5^{*}$ & $0,3-0,8$ \\
Lagarto & 76,81 & 16,62 & $4,3^{*}$ & $3,1-5,4$ \\
Nossa Senhora da Glória & 93,81 & 5,46 & 1,0 & $-0,3-2,4$ \\
Propriá & 99,11 & 1,38 & 0,1 & $-0,4-0,6$ \\
\hline
\end{tabular}

Nota: IIT: Intervalo Interquartil; APC: annual percent change; IC95\%: intervalo de confiança de 95\%; *resultados com significância estatística. Fonte: Autores. 


\subsection{Correlação ICSAP E AB}

$\mathrm{Na}$ análise da correlação de Spearman foi possível observar correlação positiva forte entre as taxas padronizadas de ICSAP e à cobertura de AB da região de Saúde de Estância, ou seja, com o aumento da cobertura $A B$ ao longo da década, houve um concomitante aumento das taxas ICSAP nessa região (Tabela 3). Essa relação também foi encontrada por Alves, Cavalcanti, Alves e Costa (2018), que descreveram o perfil das ICSAP nas 22 regiões de saúde do Ceará e verificaram que, em algumas regiões, a ampliação da cobertura de Equipes de Saúde da Família estava correlacionada ao aumento da proporção de ICSAP. Isso pode significar que uma ampliação da APS sem o compromisso com a resolubilidade, a qualidade e o acesso, não garante impacto direto sobre as taxas de internações sensíveis. Dessa forma, enquanto indicador de qualidade da APS, as ICSAP devem ser utilizadas com cautela, com análise conjunta de outras variáveis, como os relacionados ao paciente, à organização dos serviços, e a fatores socioeconômicos. Analisar o indicador de forma isolada com a variação da cobertura $\mathrm{AB}$ pode levar a conclusões enviesadas.

As demais regiões de saúde, bem como o Estado de Sergipe não apresentaram correlação entre as duas variáveis, podendo sugerir a interferência de outras variáveis nas internações (Tabela 3). Para esse estudo, os vieses ecológicos potenciais podem ser maiores devido às grandes diferenças nas características socioeconômicas presentes entre os municípios de uma mesma região de saúde.

Tabela 3: Correlação das taxas ICSAP em relação à cobertura AB em Sergipe e nas regiões de saúde de 2010 a 2019.

\begin{tabular}{ccc}
\hline & \multicolumn{2}{c}{ Cobertura da Atenção Básica } \\
\hline Estado de Sergipe & $-0,006$ & $\mathrm{p}$ \\
Regiões de Saúde & & 0,987 \\
Aracaju & 0,055 & 0,881 \\
Nossa Senhora do Socorro & $-0,067$ & 0,855 \\
Estância & 0,782 & $0,008^{*}$ \\
Itabaiana & 0,285 & 0,425 \\
Lagarto & $-0,297$ & 0,405 \\
Nossa Senhora da Glória & $-0,491$ & 0,150 \\
Propriá & 0,103 & 0,777 \\
\hline
\end{tabular}

Nota: $\mathrm{r}$ - coeficiente de correlação, *resultados com significância estatística.

Fonte: Autores.

Outros aspectos socioeconômicos também podem indicar barreiras de acesso dentro de um sistema de saúde e interferir nas taxas de ICSAP. Em alguns estudos, populações com menor renda foram associadas a taxas mais elevadas de ICSAP, assim como foi encontrado associações significativas entre menor escolaridade e maior incidência de hospitalizações. Apesar de não ter sido o escopo desse trabalho, monitorar as desigualdades socioeconômicas e entender seus impactos no 
sistema de saúde, pode contribuir para diminuir as hospitalizações, bem como efetivar os benefícios proporcionados por uma AB de qualidade (Wallar, de Prophetis, \& Rosella, 2020).

Outra estratégia para entender melhor a influência da $\mathrm{AB}$ nas hospitalizações evitáveis seria estratificar os resultados por grupos de idade e/ou por condição clínica, como foi observado nos estudos de Luz, Junger e Cavalini (2010) e Guanais e Macinko (2009), onde o efeito da maior cobertura da ESF diminuiu hospitalizações apenas em mulheres para diabetes e doenças cardiovasculares. Esses achados aliados ao maior contato das mulheres na AB, reforçam a necessidade de melhorar o acesso dos homens à $\mathrm{AB}$, para que também possam usufruir dos benefícios do uso da rede, ajudando a reduzir o número de internações por complicações de doenças crônicas (Mendonça, Harzheim, Duncan, Nunes, \& Leyh, 2012). Também foi observado, no estudo de Guimarães, Oliveira e Bohland (2020), que as ICSAP reduziram 134,9\% no grupo de adolescentes residentes de Sergipe, para ambos os sexos, considerando os dois períodos do estudo (2002 a 2004 e 2010 a 2012).

Aliada a essa perspectiva, o estudo de Cavalcanti et al (2021) evidenciou altas taxas de internações em idosos, no período de 2015 a 2019, no município de Estância. Isso pode demonstrar que algumas faixas etárias possuem mais barreiras do que outras ao acesso à saúde, causando impactos no aumento das internações. Essa vulnerabilidade dos idosos se estende desde à complexidade de locomoção até à fragilidade socioeconômica e à baixa escolaridade, reunindo diversos fatores que dificultam o acesso desse grupo à APS.

Outro fator relevante entre a correlação da cobertura e as ICSAP é a ausência dos dados de internações antes da cobertura maciça da $\mathrm{AB}$ no Estado, dificultando a comparação mais fidedigna dos dados. Uma alta cobertura já encontrada nessa última década pode explicar a estabilidade dos dados, já que a extinção das condições sensíveis não é algo esperado no âmbito atual da saúde. Soares e Ramos (2020) ratificam que essa questão pode estar conectada com o fato da AB apresentar valores mais estáveis nos últimos anos, visto que o seu crescimento acentuado ocorreu em momentos anteriores.

Além disso, a qualidade e a disponibilidade da $\mathrm{AB}$ são outros aspectos que devem ser explorados, tal como a presença de agentes comunitários de saúde, a colocação do cuidado geograficamente próximo a quem o utiliza, bem como continuidade do mesmo médico na equipe. Todos esses fatores são apontados como importantes para a redução das internações por condições sensíveis em uma região (Mendonça, et al., 2012). Por fim, é importante ressaltar a discrepância entre a estimativa de cobertura populacional de saúde da família e a real cobertura da população devidamente cadastrada em uma lista atribuída a um profissional ou a uma equipe, podendo ser um fator de confusão para a análise dos dados (Gomes, Gutiérrez, \& Soranz, 2020).

\subsection{PMAQ}

Em relação à análise do PMAQ - utilizando o teste ANOVA, seguido pelo pós teste de Bonferroni -, foi identificado que os municípios que tiveram menos equipes aderentes ao programa obtiveram maiores taxas de ICSAP, no período de 2010 a 2019, quando comparados àqueles com 50\% ou mais equipes participantes. O grupo em que todas as equipes participaram do PMAQ apresentou uma diferença de 6.629 nas taxas de ICSAP em relação ao grupo com menos de 50\% de participação, enquanto o grupo intermediário apresentou uma diferença de 6.371 com esse mesmo grupo. (Figura 1). Em um estudo de abrangência nacional, também analisando a porcentagem de equipes totais participantes do PMAQ, foi verificado que a implantação do programa foi associada a uma redução nas hospitalizações evitáveis durante as três rodadas do programa (2011-2018), sendo a participação de 89,5\% de equipes no PMAQ responsável por reduzir 3\% da taxa ICSAP, ou aproximadamente 60.829 internações em 2018 (Russo, et al., 2021). 
Figura 1: Taxas de ICSAP dos diferentes grupos participantes do segundo ciclo do PMAQ ao longo dos anos 2010 a 2019. Realizado teste ANOVA, seguido pelo pós-teste de Bonferroni.

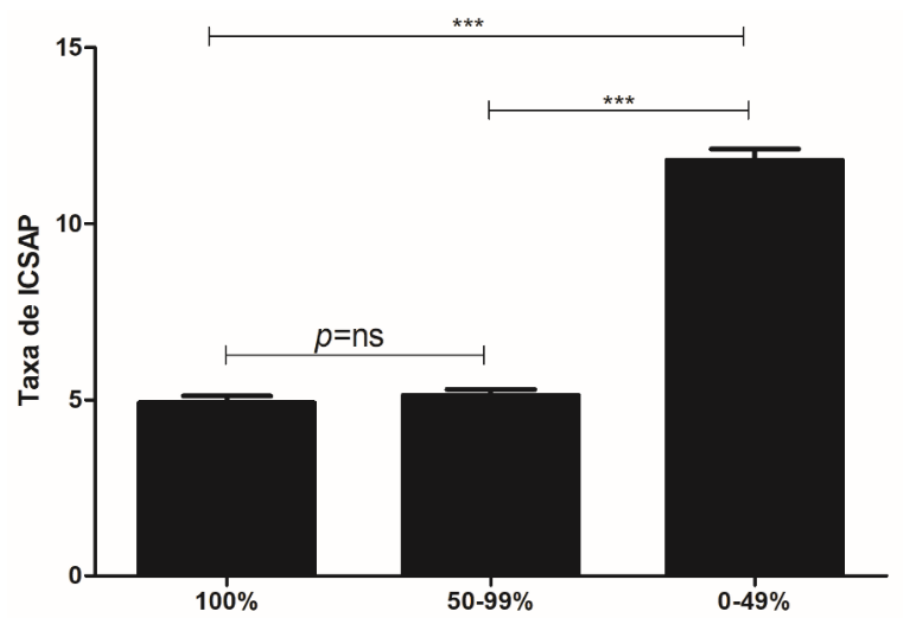

Nota: *resultados com significância estatística, ns: resultado não significativo.

Fonte: Autores

Em relação a variação da taxa ICSAP ao longo dos anos, foi observado uma estabilidade em todos os grupos. Isso pode demonstrar que, apesar da crise financeira ter atingido o país em meados do período estudado, a participação no PMAQ pode ter contribuído para a estagnação dessas internações (Figura 2). No estudo de Soares e Ramos (2020), a região Nordeste apresentou um aumento abrupto nas ICSAP nos municípios que não aderiram ao programa a partir do segundo ciclo do PMAQ, parecendo ser a região que mais se beneficiou com esse programa. Além disso, nesse mesmo estudo, todas as regiões brasileiras apresentaram taxas totais mais altas nos municípios que não aderiram ao PMAQ, de 2010 a 2014 . Esses resultados podem estar relacionados a uma maior capacidade de planejamento das equipes participantes, com busca contínua do aperfeiçoamento de ações programáticas e uma melhor gestão do serviço. 
Figura 2: Variação das taxas de ICSAP, ao longo de 2010 a 2019, nos três grupos participantes do segundo PMAQ. Realizado teste ANOVA, seguido pelo pós-teste de Bonferroni.
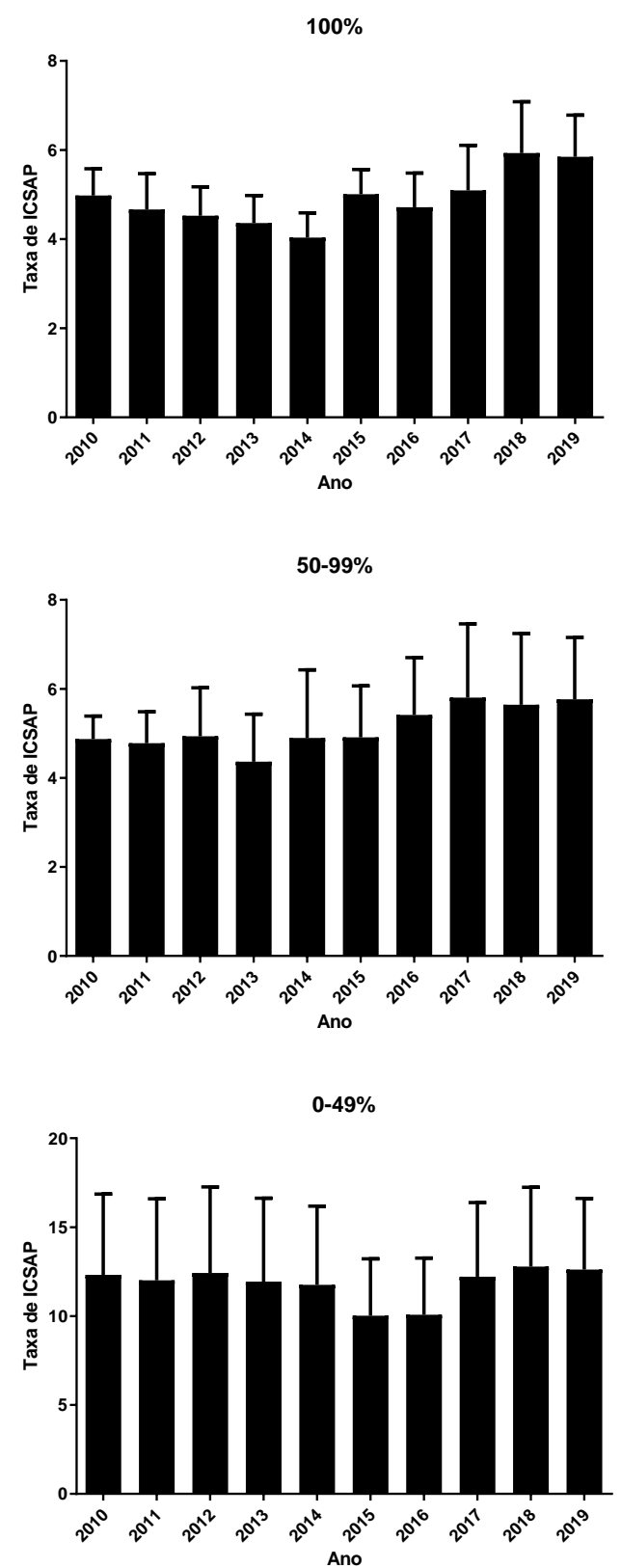

Fonte: Autores.

Também foi utilizada a estratégia de examinar o repasse financeiro mensal que cada município recebeu com o PMAQ (Figura 3). Outros estudos já analisaram a relação entre pagamento por desempenho e taxas de ICSAP e apresentaram resultados heterogêneos. Um estudo inglês mostrou uma redução nas admissões por condições influenciadas pela qualidade da atenção ambulatorial após a introdução desse esquema de pagamento (Harrison, et al., 2014). Entretanto, assim como demonstrado em estudo português, nossa relação não apresentou significância estatística entre as duas variáveis Dimitrovová, Perelman, \& Serrano-Alarcón, 2020). Apesar disso, é notório uma tendência de maiores taxas de ICSAP no grupo de municípios que tiveram um repasse financeiro menor. Isso pode significar tanto uma baixa participação de equipes disponíveis para um determinado número de população ou até mesmo uma qualidade menor dessa $\mathrm{AB}$, já que as menores notas obtidas 
irão refletir em um repasse financeiro menor. Um fator que pode ter limitado nossa análise é a mudança de padrão de pagamento a partir do terceiro ciclo do PMAQ, que teve sua primeira lista de certificação em setembro de 2018.

Figura 3: Variação das taxas de ICSAP, ao longo de 2010 a 2019, em relação ao repasse financeiro do PMAQ. Realizado teste ANOVA, seguido pelo pós-teste de Bonferroni

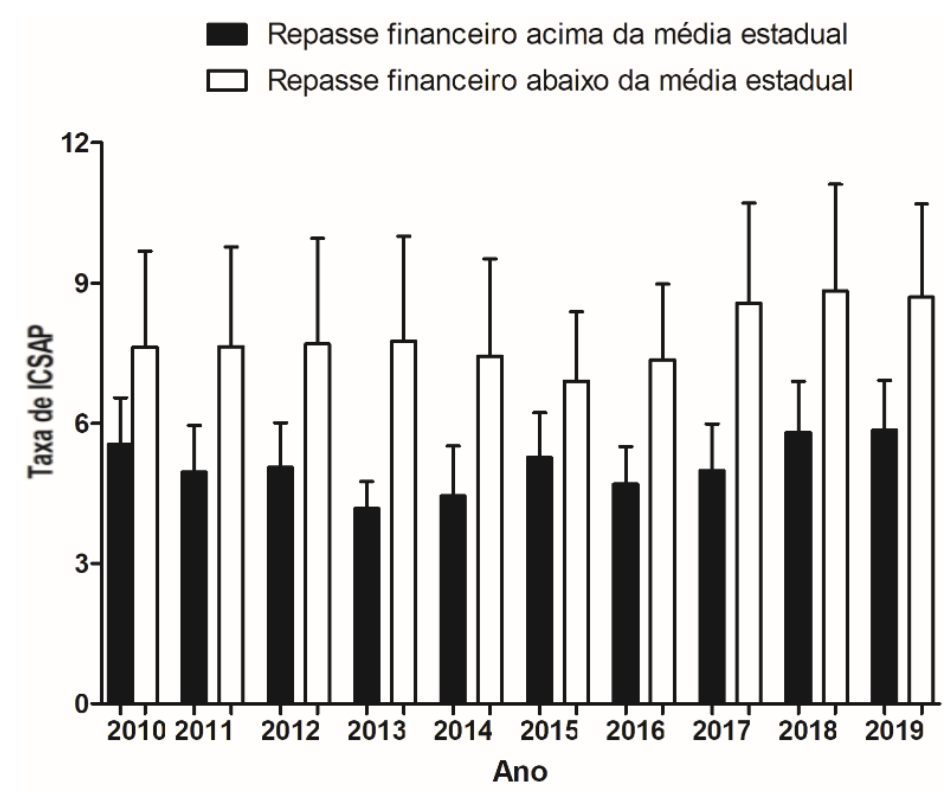

Fonte: Autores.

\section{Conclusão}

Os resultados apresentados por este estudo evidenciaram que o Estado de Sergipe apresentou uma estabilidade das ICSAP. Como indicador da qualidade da AB, o ICSAP deve ser utilizado concomitante a uma avaliação de outros aspectos histórico-estruturais, já que apresenta uma incapacidade de considerar a $\mathrm{AB}$ como único fator envolvido para seus valores. $\mathrm{A}$ análise das ICSAP desde a implantação da AB pode ser uma forma de medir os efeitos de forma mais criteriosa a médio e longo prazos, já que o período do estudo foi posterior ao investimento maciço da expansão da cobertura $\mathrm{AB}$. Com seu uso mais amplo, os bancos de dados podem se beneficiar, tornando-se mais densos e completos, além de direcionar a gestão em saúde com a exposição de diferentes fatores na redução das internações.

O monitoramento dessas internações, por faixa etária e pelos grupos de doenças presentes na lista, também pode servir para apontar prioridades de investimentos nos serviços de saúde, visando determinar quais barreira de acesso à APS estão ocorrendo em um determinado local, assim como determinar quais fatores socioeconômicos extrapolam a ação da AB.

Além disso, a partir dos resultados obtidos, foi possível concluir que o PMAQ atingiu o seu objetivo em aumentar o acesso e a qualidade da AB no Estado de Sergipe, mostrando que foi capaz de proporcionar estagnação das taxas, apesar de um cenário financeiro e político de instabilidade. Cabe destacar as limitações referentes à análise somente do segundo ciclo, exigindo cautela ao analisar os efeitos do PMAQ nas ICSAP. Nesse contexto, este artigo surge como um importante informativo sobre as políticas públicas de saúde no Brasil e, também, como um meio de discussão de um dos programas de incentivo da AB: o PMAQ.

Sugere-se mais estudos abordando hospitalizações sensíveis ao nível primário, principalmente no Estado de Sergipe, tendo em vista sua magnitude em relação ao impacto no planejamento e na gestão em saúde, aos avanços das políticas públicas 
brasileiras e na humanização da assistência. Uma maior vigilância e consideração desses dados poderia influenciar as equipes a aperfeiçoarem a atenção à saúde e, consequentemente, diminuírem as ICSAP de forma mais expressiva. Como o PMAQ foi descontinuado pelo MS, em dezembro de 2019, novas formas de financiamento da AB foram estabelecidas. Com isso, nossos resultados podem representar meios de debate sobre os ajustes dos esquemas de pagamento por desempenho no cenário do Brasil, servindo de comparação para novos estudos sobre seu impacto no acesso e na qualidade da AB.

\section{Referências}

Agabiti, N., Pirani, M., Schifano, P., Cesaroni, G., Davoli, M., Bisanti, L., Caranci, N., Costa, G., Forastiere, F., Marinacci, C., Russo, A., Spadea, T., Perucci, C. A., \& Italian Study Group on Inequalities in Health Care (2009). Income level and chronic ambulatory care sensitive conditions in adults: a multicity population-based study in Italy. BMC public health, 9, 457. https://doi.org/10.1186/1471-2458-9-457

Ahmad, O. B., Boschi-pinto, C., \& Lopez, A. D. (2001). Age standardization of rates: a new WHO standard. GPE Discussion Paper Series, (31), 10-12.

Alfradique, M. E., Bonolo, P. de F., Dourado, I., Lima-Costa, M. F., Macinko, J., Mendonça, C. S., Oliveira, V. B., Sampaio, L. F. R., Simoni, C. de, \& Turci, M. A. (2009) Ambulatory care sensitive hospitalizations: Elaboration of brazilian list as a tool for measuring health system performance (project ICSAP Brazil). Cadernos de Saude Publica, 25 (6), 1337-1349. https://doi.org/10.1590/S0102-311X2009000600016

Alves, J. W. dos S., Cavalcanti, C. G. C. S., Alves, R. S. M., \& Costa, P. C. da (2018) Internações por Condições Sensíveis à Atenção Primária no estado do Ceará, 2010-2014. Saúde em Debate, 42 (spe4), 223-235. https://doi.org/10.1590/0103-11042018S418.

Bastos, M. L., Menzies, D., Hone, T., Dehghani, K., \& Trajman, A. (2017). The impact of the Brazilian family health on selected primary care sensitive conditions: A systematic review. PLoS ONE, 12 (8), 1-14. https://doi.org/10.1371/journal.pone.0182336

Billings, J., Zeitel, L., Lukomnik, J., Carey, T. S., Blank, A. E., \& Newman, L. (1993) Impact of socioeconomic status on hospital use in New York City. Health Affairs. 12 (1), 162-173. https://doi.org/10.1377/hlthaff.12.1.162

Cavalcanti, S., Morais, C., Feitosa, S., Mirelle, D., Santos, S., Dantas Barros, F., \& Carvalho, A.C.A.(2021) Internações por condições sensiveis á atenção primária: município do nordeste do Brasil / Hospitalizations for conditions sensitive to primary care: a city in the northeast of Brazil. Brazilian Journal of Health Review, 4 (2), 4298-4310. https://doi.org/10.34119/bjhrv4n2-026

Dimitrovová, K., Perelman, J., \& Serrano-Alarcón, M. (2020) Effect of a national primary care reform on avoidable hospital admissions (2000-2015): A difference-in-difference analysis. Social Science \& Medicine, 252, 112908. https://doi.org/10.1016/j.socscimed.2020.112908

Guanais, F., \& Macinko, J. (2009) Primary care and avoidable hospitalizations: evidence from Brazil. The Journal of ambulatory care management, 32 (2), 115-122. https://doi.org/10.1097/JAC.0b013e31819942e51

Gomes, C. B. E S., Gutiérrez, A. C., \& Soranz, D. (2020) Política Nacional de Atenção Básica de 2017: análise da composição das equipes e cobertura nacional da Saúde da Família. Ciência \& Saúde Coletiva, 25 (4), 1327-1338. https://doi.org/10.1590/1413-81232020254.31512019

Governo do Estado de Sergipe. Secretaria de Estado da Saúde (2016). Plano Estadual de Saúde - Vigência 2016 a 2019. Recuperado em 04 janeiro 2020 , de https://www.conass.org.br/pdf/planos-estaduais-de-saude/SE_Plano\%20Estadual\%20de\%20Saude\%202016\%20-\%202019\%20SERGIPE.pdf.

Guimarães, N. M., Oliveira, E. R., \& Bohland, A. K. (2020) Hospital Admissions of Adolescents in Sergipe, from 2002 to 2012. Revista Paulista de Pediatria, 38 (25). https://doi.org/10.1590/1984-0462/2020/38/2018181

Harrison, M. J., Dusheiko, M., Sutton, M., Gravelle, H., Doran, T., \& Roland, M. (2014) Effect of a national primary care pay for performance scheme on emergency hospital admissions for ambulatory care sensitive conditions: controlled longitudinal study. BMJ, 349 (g6423). https://doi.org/10.1136/bmj.g6423

Justo, C., Bastos, K. A., \& Nedel, F. B.(2020). Public policies reected on hospitalizations for ambulatory care sensitive conditions: an ecological study in Sergipe-Brazil, 2008-2017. Research Gate. https://doi.org/10.21203/rs.3.rs-59466/v1.

Kim, A. M., Park, J. H., Yoon, T. H., \& Kim, Y. (2019). Hospitalizations for ambulatory care sensitive conditions as an indicator of access to primary care and excess of bed supply. BMC Health Services Research, 19 (1), 1-7. https://doi.org/10.1186/s12913-019-4098-x

Luz, C. De C., Junger, W. L. \& Cavalini, L. T. (2010) Analysis of pre-hospital care for stroke and myocardial infarction in the elderly population of minas gerais. Revista da Associação Médica Brasileira, 56 (4), 452-457. https://doi.org/10.1590/S0104-42302010000400019

Macinko, J., Harris, M. J. \& Rocha, M. G. (2017) Brazil's national program for improving primary care access and quality (PMAQ) fulfilling the potential of the world's largest payment for performance system in primary care. Journal of Ambulatory Care Management, 40 (2), S4-S11. https://doi.org/10.1097/JAC.0000000000000189.

Macinko, J., de Oliveira, V. B. , Turci, M. A., Guanais, F. C., Bonolo, P. F. \& Lima-Costa, M. F. (2011) The Influence of Primary Care and Hospital Supply on Ambulatory Care-Sensitive Hospitalizations Among Adults in Brazil. American Journal of Public Health, 101(10),1963-70. https://doi.org/10.2105/AJPH.2010.198887

Mendonça, C. S., Harzheim, E., Duncan, B. B., Nunes, L. N., \& Leyh, W. (2012) Trends in hospitalizations for primary care sensitive conditions following the implementation of Family Health Teams in Belo Horizonte, Brazil. Health Policy and Planning, 27 (4), 348-355. https://doi.org/10.1093/heapol/czr043

Pedraza, D. F. \& de Araujo, E. M. N. (2017) Internações das crianças brasileiras menores de cinco anos: revisão sistemática da literatura. Epidemiologia e 
Research, Society and Development, v. 11, n. 1, e42211125099, 2022

(CC BY 4.0) | ISSN 2525-3409 | DOI: http://dx.doi.org/10.33448/rsd-v11i1.25099

servicos de saude, 26 (1), 169-182. https://doi.org/10.5123/S1679-49742017000100018

Pinto, L. F., Mendonça, C. S., Rehem, T. C. M. S. B., \& Stelet, B. (2019) Hospitalisations due to ambulatory care sensitive conditions (Acsc) between 2009 and 2018 in brazil's federal district as compared with other state capitals. Ciencia e Saude Coletiva, 24 (6), 2105-2114. https://doi.org/10.1590/141381232018246.08582019

Portaria $n^{\circ} 1.654$ (19, julho, 2011). Institui, no âmbito do Sistema Único de Saúde, o Programa Nacional de Melhoria do Acesso e da Qualidade da Atenção Básica (PMAQ-AB) e o Incentivo Financeiro do PMAQ-AB, denominado Componente de Qualidade do Piso de Atenção Básica Variável - PAB Variável.. Diário Oficial da União $\mathrm{n}^{\circ} 138 \quad$ (20, julho, 2011). Brasilia, DF: Ministério da Saúde. http://189.28.128.100/dab/docs/portaldab/documentos/pmaq/prt_1654_19_07_2011.pdf

Rasella, D., Harhay, M. O., Pamponet, M. L., Aquino, R., \& Barreto, M. L. (2014) Impact of primary health care on mortality from heart and cerebrovascular diseases in Brazil: A nationwide analysis of longitudinal data. BMJ (Online), 349, g4014. https://doi.org/10.1136/bmj.g4014

Rasmussen, S. R., Kilsmark, J., Hvenegaard, A., Søgaard, J., Thomsen, J. L., Engberg, M. \& Lauritzen, T. (2007) Preventive health screenings and health consultations in primary care increase life expectancy without increasing costs. Scandinavian Journal of Public Health, 35 (4), 365-372. https://doi.org/10.1080/14034940701219642.

Ribeiro, M. G. C., Araujo Filho, A. C. A., \& da Rocha, S. S. (2019) Hospitalizações por condições sensíveis à atenção primária em crianças do Nordeste Brasileiro. Revista Brasileira de Saúde Materno Infantil, 19 (2), 491-498. https://doi.org/10.1590/1806-93042019000200013

Rosano, A., Loha, C. A., Falvo, R., Zee, J. Van Der, Ricciardi, W., Guasticchi, G., \& de Belvis, A.G. (2012) The relationship between avoidable hospitalization and accessibility to primary care: a systematic review. European Journal of Public Health, 23 (3), 356-360. https://doi.org/10.1093/eurpub/cks053.

Russo, L. X., Powell-Jackson, T., Barreto, J. O. M., Borghi, J., Kovacs, R., Gurgel Junior, G. D., Gomes, L. B., Sampaio, J., Shimizu, H. E., Sousa, A. N. A., Bezerra, A. F. B., Stein, A. T., \& Silva, E. N. (2021). Pay for performance in primary care: the contribution of the Programme for Improving Access and Quality of Primary Care (PMAQ) on avoidable hospitalisations in Brazil, 2009-2018. BMJ Global Health, 6, e005429. http://dx.doi.org/10.1136/bmjgh-2021005429

Sala, A. \& Mendes, J. D. V. (2011). Perfil de indicadores da atenção primária à saúde no estado de São Paulo: retrospectiva de 10 anos. Saúde e Sociedade, 20 (4), 912-926. https://doi.org/10.1590/S0104-12902011000400009

Soares, C. \& Ramos, M. (2020) An evaluation of PMAQ-AB effects on hospitalization for conditions susceptible to Primary Care. Saúde em Debate, 44 (126), 708-724. https://doi.org/10.1590/0103-1104202012609

Wallar, L. E., de Prophetis, E., \& Rosella, L. C. (2020) Socioeconomic inequalities in hospitalizations for chronic ambulatory care sensitive conditions: A systematic review of peer-reviewed literature, 1990-2018. International Journal for Equity in Health, 19 (60). https://doi.org/10.1186/s12939-020-01160-0 